\section{La drosophile comme nouveau modèle d'étude des leucémies}

Dani Osman, Vanessa Gobert, Marc Haenlin, Lucas Waltzer
Université de Toulouse, UPS,

Centre de biologie du développement (CBD), Bâtiment 4R3, 118, route de Narbonne,

31062 Toulouse, France ;

CNRS, CBD UMR5547, 31062 Toulouse, France waltzer@cict.fr

modèles ont permis de montrer qu'AMLIદTO inhibe la différenciation myéloïde et promeut la conservation d'un nombre élevé de progéniteurs hématopoiétiques. Cependant, si la translocation $\mathrm{t}(8 ; 21)$ semble être un élément initiateur, des mutations secondaires (conduisant par exemple à une activation constitutive de Ras ou de Flt3) sont requises pour que les cellules exprimant AML1-\&TO deviennent leucémiques. En parallèle, des analyses transcriptomiques et plus récemment par ChIP-chip ont permis d'identifier des gènes dérégulés par AML1-દTO et de mieux comprendre les voies de signalisation en aval de cet oncogène. Les facteurs qui modulent l'activité d'AML1हTO restent en revanche pour la plupart inconnus.

\section{Modéliser le rôle d'AML1-ETO}

chez la drosophile

L'identification des gènes qui interagissent fonctionnellement avec AMLIETO est essentielle, mais sa réalisation demeure ardue dans les systèmes mammifères. Afin d'établir un modèle d'étude d'AML1-દTO qui se prête à une analyse génétique de son activité, nous

Figure 1. Représentation schématique d'AML1,

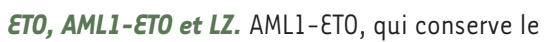
domaine RUNT de liaison à l'ADN d'AMLI, interfère avec la régulation transcriptionnelle des cibles d'AMLl et agit au moins en partie comme dominant négatif. Le domaine RUNT de LZ présente $71 \%$ d'identité avec celui d'AMLl. Les différents domaines structuraux sont indiqués. WRPY : domaine d'interaction avec les corépresseurs de la famille GRO/TLE. NHR 1-4: nervy homology regions 1-4. PEST : domaine PEST. 


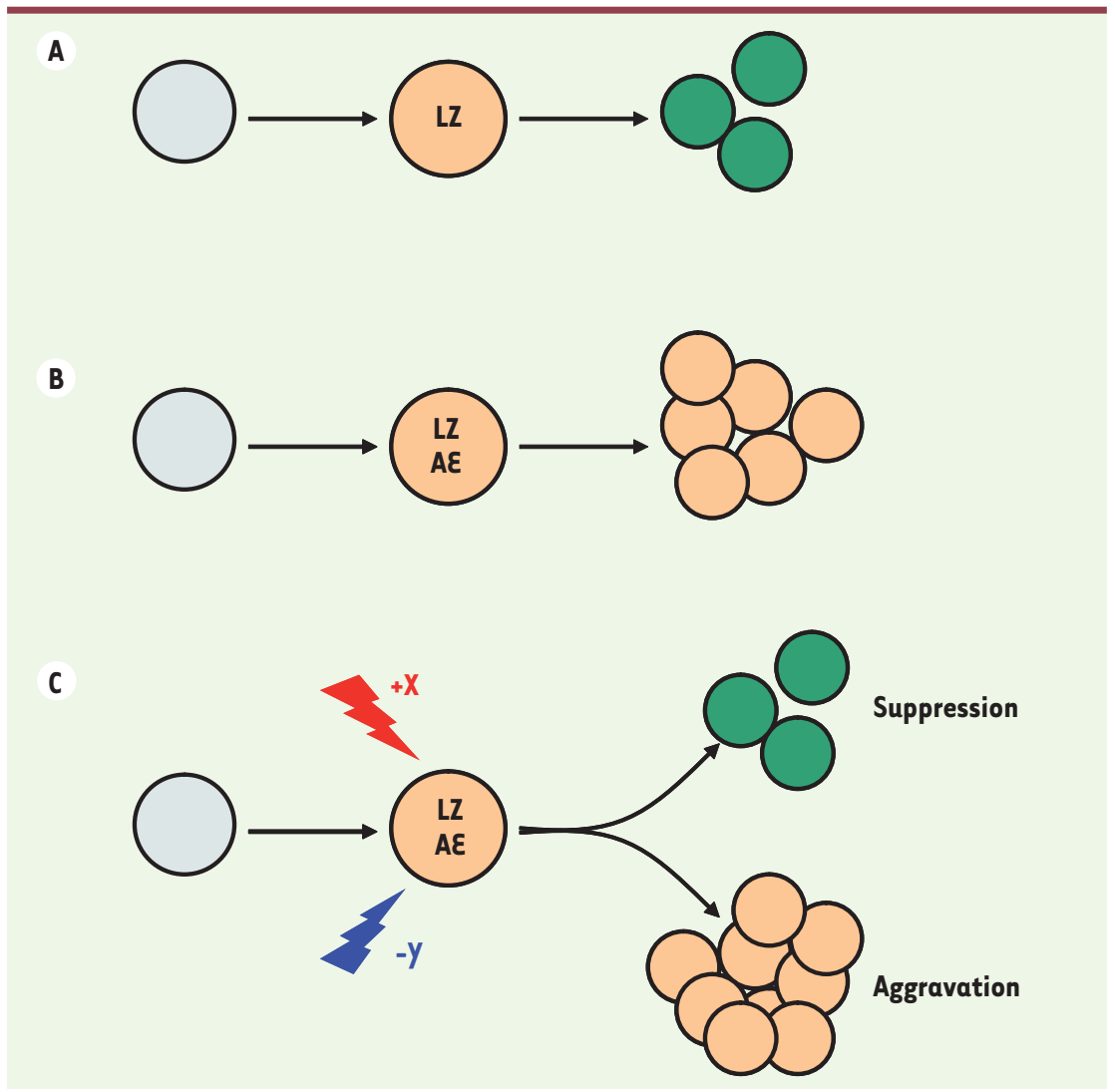

avons caractérisé l'effet de l'expression d'AMLI-ETO dans le système hématopoiétique de la drosophile. En effet, malgré la distance phylogénétique qui sépare ces deux espèces, différents aspects du développement des cellules hématopoïétiques ont été conservés de la drosophile à l'homme [4]. En particulier, la drosophile possède deux classes principales de cellules sanguines (ou hémocytes), les plasmatocytes et les cellules à cristaux, qui ressemblent structurellement et fonctionnellement aux cellules myéloïdes des vertébrés. Le développement des cellules à cristaux dépend en particulier du facteur de transcription de type RUNX Lozenge (LZ) [5] (Figure 1), qui est exprimé dans une sous-population de progéniteurs hématopoïétiques dont il va promouvoir la différenciation [6, 7] (Figure 2). De façon intéressante, nous avons observé que l'expression d'AMLI-ETO dans les cellules sanguines de la drosophile induit des phénotypes de type «pré-
- Ce stade se situe entre la phase larvaire et la phase adu Le stade pupal est marqué par l'épaississement des téguments extérieurs, par l'émergence des cornes antérieures; à l'issue de se stade s'opère la métamorphose au cours de laquelle la plupart des tissus larvaires sont détruits et remplacés par les tissus adultes.
Figure 2. Phénotypes induits par l'expression d'AML1-ETO (AE) dans les cellules sanguines de la drosophile exprimant LZ. A. Le facteur RUNXLZ est exprimé dans les progéniteurs (orange) des cellules à cristaux dont il induit la différenciation (vert). B. L'expression d'AMLIETO dans les cellules $\mathrm{LZ}^{+}$empêche leur différenciation et promeut leur expansion. C. Dans des conditions de crible génétique (induction de l'expression ectopique d'un gène $X$ ou de la perte de fonction d'un gène $y$ dans les cellules $L Z^{+}$), les phénotypes induits par AMLI-ETO peuvent être soit supprimés soit aggravés.

I'ARN, une dizaine de suppresseurs de la létalité induits par AMLI-ETO a été identifiée parmi lesquels une calpaïne [8]. Les calpaïnes forment une famille conservée de protéases dépendantes du $\mathrm{Ca}^{2+}$ qui régulent des aspects fondamentaux de la physiologie cellulaire tels que la migration ou l'apoptose, mais leur rôle dans le développement de leucémies n’avait jamais été exploré $[9,12]$. II s'avère que la perte de fonction du gène calpain $B$ supprime non seulement la létalité mais aussi les phénotypes préleucémiques induits par l'expression d'AMLI-ETO chez la drosophile. En outre, nous avons pu montrer que l'inhibition de l'activité des calpaines chez l'homme réduit fortement la croissance et la clonogénicité de cellules leucémiques exprimant AML1-ETO. Ainsi, ces résultats suggèrent que les calpaïnes sont requises pour l'activité oncogénique d'AMLIETO de la drosophile à l'homme.

Cette étude établit le premier modèle «drosophile» d'étude de la leucémogenèse associée à la translocation $\mathrm{t}(8 ; 21)$ et ouvre de nombreuses perspectives. Concernant les calpaïnes, le mécanisme moléculaire par lequel ces enzymes participent à l'activité d'AML1-ETO reste à établir. Les calpaïnes clivent généralement leur substrat de manière limitée et génèrent fréquemment des fragments stables possédant des activités différentes de celles du substrat intact. Chez 
la drosophile comme chez l'homme, l'inhibition des calpaïnes induit une déstabilisation d'AML1-ETO au niveau posttranscriptionnel [8]. II est possible que les calpaïnes agissent via les caspases et/ou le protéasome qui sont connus pour réguler la stabilité d'AML1-ETO [10]. Une autre possibilité est que AML1-عTO, qui possède deux domaines de type PEST (proline, glutamate, sérine, et thréonine) (Figure 1), également présents dans de nombreux substrats des calpaïnes, soit une cible directe de ces enzymes. D'un point de vue médical, différents inhibiteurs pharmacologiques de ces enzymes sont disponibles et suscitent beaucoup d'intérêt pour le traitement de maladies neurodégénératives de type Parkinson ou Alzheimer. Le potentiel thérapeutique de ces inhibiteurs dans le cas de leucémies aiguës pourrait donc être testé relativement rapidement.

\section{La drosophile, quelle place comme} modèle de tumorigenèse humaine?

Plus généralement, ces résultats ouvrent la voie à l'utilisation de la drosophile comme tube à essai pour identifier d'autres gènes dont les homologues humains régulent l'activité d'AMLI-ETO dans les leucémies. Les puissants outils disponibles chez la drosophile permettent de réaliser différents types de cribles génétiques (Figure 2), que ce soit en perte de fonction ou en gain de fonction. Comme l'expression d'AML1-ETO n'est pas suffisante per se pour induire des leucémies chez les mammifères [3], il serait intéressant de rechercher des enhancers d'AMLI-ETO chez la drosophile dans le but d'identifier les voies de signalisation qui coopèrent avec cet oncogène. Ainsi, on peut espérer que la drosophile permette de mettre à jour les réseaux génétiques conservés qui constituent la base de la transformation cellulaire et que ce modèle aidera à mieux comprendre le mode d'action d'AML1-ETO mais aussi d'autres oncogènes. $\diamond$

Drosophila as a new model system for leukaemia

\section{CONFLIT D'INTÉRÊTS}

Les auteurs déclarent n'avoir aucun conflit d'intérêts concernant les données publiées dans cet article.

\section{RÉFÉRENCES}

1. Niebuhr B, Fischer M, Tager M, et al. Gatekeeper function of the RUNXI transcription factor in acute leukemia. Blood Cells Mol Dis 2008; 40 : 211-8.
2. Elagib KE, Goldfarb A. Oncogenic pathways of AMLI-ETO in acute myeloid leukemia: multifaceted manipulation of marrow maturation. Cancer Lett $2007 ; 251: 179-86$

3. McCormack $\varepsilon$, Bruserud 0, Gjertsen BT. Review: genetic models of acute myeloid leukaemia. Oncogene 2008; $27: 3765-79$

4. Hartenstein V. Blood cells and blood cell development in the animal kingdom. Annu Rev Cell Dev Biol 2006 ; $22: 677-712$.

5. Lebestky T, Chang T, Hartenstein V, Banerjee U. Specification of Drosophila hematopoietic lineage by conserved transcription factors. Science 2000 ; 288: 146-9.

6. Waltzer L, Ferjoux G, Bataillé L, Haenlin M. Cooperation between the GATA and RUNX factors Serpent and Lozenge during Drosophila hematopoiesis. Embo J 2003; $22: 6516-25$.

7. Ferjoux G, Augé B, Boyer K, et al. A GATA/RUNX cisregulatory module couples Drosophila blood cell commitment and differentiation into crystal cells. Dev Biol 2007 ; 305 : 726-34.

8. Osman D, Gobert V, Ponthan F, et al. A Drosophila model identifies calpains as modulators of the human leukemogenic fusion protein AML1-ETO. Proc Natl Acad Sci USA 2009; 106 : 12043-8.

9. Croall $D E$, Ersfeld $K$. The calpains: modular designs and functional diversity. Genome Biol $2007 ; 8: 218$

10. Corsello SM, Roti C, Ross KN, et al. Identification of AMLI-ETO modulators by chemical genomics. Blood $2009 ; 113: 6193-205$.

11. Godin I, Cumano A. Hematopoietic stem cells: where do they come from at last? Med Sci (Paris) 2007 ; $23: 681-4$.

12. Baud L, Fouqueray B, Bellocq A, Peltier J. Calpains participate in inflammatory reaction development. Med Sci (Paris) 2003; 19:71-6.

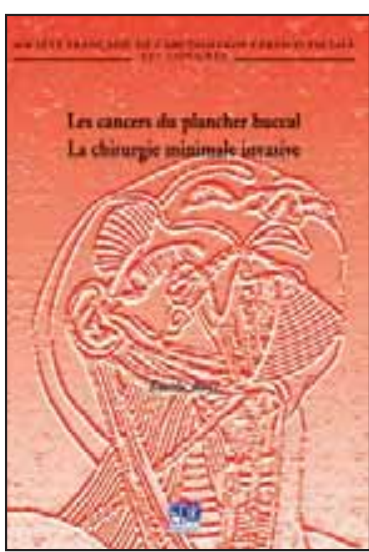

ISBN : 978-2-8425-4127-9 212 pages

\section{Bon de commande}

À retourner à EDK, 2, rue Troyon - 92316 Sèvres Cedex

Tél. : 0155641393 - Fax : 0155641394 - E-mail : edk@edk.fr

NOM : Prénom :

Adresse :

Code postal : _...… _ _ Ville :

Pays :

Fonction :

Je souhaite recevoir l'ouvrage Les cancers du plancher buccal : $35 €+3 €$ de port $=38 €$ TTC

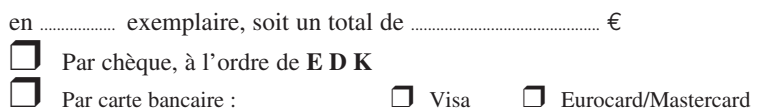

Carte $n^{\circ}+1+1+1+1+1+1+1$

Date d'expiration: $\quad$ । 1 | 1 | |

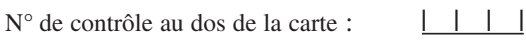

Signature : 